\title{
Age- and sex-specific reference intervals for thyroid hormones in a Chinese pediatrics: a prospective observational study of 1,279 healthy children
}

\author{
Cong Yao ${ }^{1 \#}$, Mo Wu ${ }^{2 \#}$, Mei Liu ${ }^{3 \#}$, Xiaoqian Chen ${ }^{4}$, Hongmin $\mathrm{Zhu}^{5}$, Chen Xiong ${ }^{6}$, Dan Wang ${ }^{7}$, Yun Xiang ${ }^{2}$, \\ Guori Suo ${ }^{6}$, Jun Wang ${ }^{2}$, Hong Sun ${ }^{2}$, Chunhui Yuan ${ }^{2}$, Yong Xia ${ }^{8}$
}

${ }^{1}$ Health Care Department, Wuhan Children's Hospital (Wuhan Maternal and Child Healthcare Hospital), Tongji Medical College, Huazhong University of Science \& Technology, Wuhan, China; ${ }^{2}$ Department of Laboratory Medicine, Wuhan Children's Hospital (Wuhan Maternal and Child Healthcare Hospital), Tongji Medical College, Huazhong University of Science \& Technology, Wuhan, China; ${ }^{3}$ Department of Laboratory Medicine, Wuhan Hankou Hospital, Wuhan, China; ${ }^{4}$ Department of Endocrinology, Genetics \& Metabolism, Wuhan Children's Hospital (Wuhan Maternal and Child Healthcare Hospital), Tongji Medical College, Huazhong University of Science \& Technology, Wuhan, China; ${ }^{5}$ Department of Neurology, Wuhan Children's Hospital (Wuhan Maternal and Child Healthcare Hospital), Tongji Medical College, Huazhong University of Science \& Technology, Wuhan, China; ${ }^{6}$ Shenzhen Mindray Bio-Medical Electronics Co., Ltd., Shenzhen, China; ${ }^{7}$ Department of Neonatology, Wuhan Children's Hospital (Wuhan Maternal and Child Healthcare Hospital), Tongji Medical College, Huazhong University of Science \& Technology, Wuhan, China; ${ }^{8}$ Clinical Medical Laboratory, Peking University Shenzhen Hospital, Shenzhen, China

Contributions: (I) Conception and design: H Sun, C Yuan, Y Xia; (II) Administrative support: H Sun, C Yuan, Y Xia; (III) Provision of study materials or patients: M Wu, X Chen, H Zhu, C Xiong, D Wang, Y Xiang, G Suo, J Wang; (IV) Collection and assembly of data: C Yao, M Wu, M Liu; (V) Data analysis and interpretation: C Yao, Y Xia, C Yuan; (VI) Manuscript writing: All authors; (VII) Final approval of manuscript: All authors.

\#These authors contributed equally to this work.

Correspondence to: Hong Sun; Chunhui Yuan. Department of Laboratory Medicine, Wuhan Medical and Health Center for Women and Children, Tongji Medical College, Huazhong University of Science and Technology, Wuhan 430016, China. Email: shong19@163.com; chunhuii.yuen@whu.edu.cn; Yong Xia. Clinical Medical Laboratory, Peking University Shenzhen Hospital, Shenzhen 518036, China. Email: sunmoonrain78@163.com.

Background: Thyroid hormones are essential for early neurocognitive development and growth and development in childhood and adolescence. However, the reference intervals (RIs) for thyroid hormones in Chinese pediatric individuals remain unclear. This study aimed to establish thyroid hormone RIs for a Chinese pediatric population according to appropriate age- and sex-specific partitioning.

Methods: In this prospective observational study, a total of 1,279 healthy children (singletons, aged from 1 day to 12 years) were recruited, and serum samples were analyzed on a Mindray automated chemiluminescence immunoassay analyzer CL-6000i for thyroid hormone detection, including thyroidstimulating hormone (TSH), free triiodothyronine (FT3), free thyroxine (FT4), total triiodothyronine (T3), and total thyroxine (T4). Age and sex-specific RIs were established, and the corresponding 95\% confidence intervals (CIs) were calculated in accordance with the Clinical Laboratory Standards Institute (CLSI) document C28-A3 guidelines.

Results: Quantile testing revealed that the median (P50) and RIs $\left[2.5^{\text {th }}\right.$ percentile $(\mathrm{P} 2.5)-97.5^{\text {th }}$ percentile (P97.5)] for TSH, FT3, T3, and T4 of males differed significantly from those of females $(\mathrm{P}<0.05)$, except for FT4 (P=0.483). For FT3 and T3, the RIs of males were higher than that of females, and the RI of T4 for males was narrower and higher than that of females [P2.5-P97.5: 72.33-171.60 vs. 72.31-176.27 nmol/L; P50: 116.75 vs. 113.47 nmol/L, P=0.011]. RIs for TSH, FT3, FT4, T3, and T4 showed sex- and agespecific properties and displayed a wide variation during the first month of life but gradually narrowed and concentrated with increasing age. In addition, RIs of TSH, FT3, FT4, and T3 in males differed significantly from females in the first month of life (TSH: 1.46-10.87 vs. 1.08-11.35 mIU/L; FT3: 2.96-7.08 vs. 2.35-7.27 pmol/L, FT4: 13.34-28.65 vs. 13.82-31.83 pmol/L; T3: 0.83-2.33 vs. 0.72-2.46 nmol/L). The RI of T4 also exhibited a difference between males and females in the 9- to 12-year age group (59.31-150.72 vs. 63.29-146.94 pmol/L for males and females, respectively). 
Conclusions: Pediatric RIs of thyroid hormones display age- and sex-specific trends. The RIs established in this study will improve the accuracy of TSH assay result interpretations and clinical decision-making in clinical laboratories that utilize the Mindray analytical platform.

Keywords: Thyroid function; thyroid hormone; reference interval (RI); children

Submitted Aug 03, 2021. Accepted for publication Sep 16, 2021.

doi: $10.21037 / \mathrm{tp}-21-389$

View this article at: https://dx.doi.org/10.21037/tp-21-389

\section{Introduction}

Thyroid follicle epithelial cells are responsible for synthesizing, storing, and secreting thyroid hormones, which are critical for early neurocognitive development and growth and development throughout childhood and adolescence (1). The secretion of thyroid hormones is mainly regulated by the feedback regulation of the hypothalamus-pituitary-thyroid axis. This negative feedback cycle keeps the concentration of thyroid hormones in a stable and circulating state to maintain the normal function of the corresponding physiological processes (2).

Abnormal thyroid function is associated with a wide range of adverse child development outcomes (3). Hypothyroidism in children is associated with mental retardation, short stature, and delayed skeletal maturation and puberty (4). In contrast, hyperthyroidism in children is associated with hyperactivity, tachycardia, irritability, poor school performance, and many other abnormal manifestations (5). Newborns with congenital hypothyroidism $(\mathrm{CH})$ who underwent early treatment within 2 weeks of diagnosis showed no significant differences in nervous system development and intelligence levels compared with normal children (6). However, failure to promptly detect and commence appropriate thyroid treatment during the neonatal period can lead to low intelligence in children, affecting their quality of life (7). The prevalence of hypothyroidism and hyperthyroidism in newborns/ childhood is an estimated $1 \%$ to $2 \%$ and 0.1 to $3 / 100,000$, respectively (3). And more importantly, thyroid dysfunction is further frequently associated with heart failure and infection $(8,9)$. Therefore, early and objective evaluation of thyroid dysfunction in children is critical.

The thyroid-stimulating hormone (TSH) assay plays a crucial role in the evaluation of thyroid function. It includes the combined detection of serum TSH, free triiodothyronine (FT3), free thyroxine (FT4), total triiodothyronine (T3), and total thyroxine (T4) levels (10).
Despite its importance, the accuracy of the TSH assay interpretation in pediatric populations is often limited by the lack of appropriate reference intervals (RIs). The guidelines of the European Thyroid Association for the management of subclinical hypothyroidism in children recommend the use of age-related normative values (11). Childhood and adolescence are accompanied by many dynamic physiological changes that parallel growth and development, and which indicated that the establishment of accurate pediatric RIs for the TSH assay requires a sufficiently large, healthy pediatric population to allow appropriate age- and sex-specific partitioning. As a result, many laboratories continue to use unverified RIs that established by various indirect methods, like the Hoffman, Bhattacharya and Arzideh methods, using inpatient data collected for the clinical pediatric care of thyroid diseases due to the immense resources required and limited resampling opportunities (12).

To date, multiple international researchers or organizations have established RIs of thyroid hormones in pediatric populations (12-17). For example, the Canadian Laboratory Initiative on Pediatric Reference Intervals (CALIPER, https://www.caliperdatabase.ca/) has tested serum samples from 741 healthy children and adolescents ( 1 day to $<19$ years) on the Roche Cobas 8000 e602 immunoassay analyzer to establish age- and sex-specific RIs for thyroid hormones (12). However, RIs of thyroid hormones can be influenced by age, Tanner stage, ethnicity, anthropometric characteristics, iodine intake, detection methodology, or other determinants $(15,18)$. Therefore, there is considerable between-study heterogeneity in the RIs of thyroid hormones in pediatric populations. In addition, most studies of thyroid hormone RIs have focused on TSH and FT4, and information concerning FT3, T3, and T4 is sparse (19-21).

The sex-specific and gestation-specific RIs of thyroid hormones have been established in Chinese adult populations (22-25). Therefore, the present study aimed 
to evaluate the distribution of thyroid hormones and to establish age- and sex-specific RIs for a Chinese pediatric population. To achieve this goal, serum TSH, FT4, FT3, T3, and T4 levels in 1,279 healthy Chinese children (0-12 years) were directly tested on the Mindray automated chemiluminescence immunoassay analyzer CL-6000i, and RIs were established according to the Clinical Laboratory Standards Institute (CLSI) document C28-A3 guidelines. We present the following article in accordance with the STROBE reporting checklist (available at https://dx.doi. org/10.21037/tp-21-389).

\section{Methods}

\section{Ethical approval}

This prospective observational study was performed in accordance with the principles of the Declaration of Helsinki (as revised in 2013) and was approved by the Ethics Committee and Institutional Review Board of the Wuhan Children's Hospital (Wuhan Maternal and Child Healthcare Hospital, 2020R052). Written informed consent was obtained from all pediatric participants (provided by at least a parent or guardian) during their physical examination.

\section{Population for analysis}

The participants consisted of 1,279 children who visited the physical examination center of Wuhan Children's Hospital from September 1, 2020, to March 17, 2021. According to age distribution, the reference population was categorized into the following six age groups: 1-31 (d), 1-12 (m), 12-36 (m), 36-72 (m), 72-108 (m), and 108-144 (m). The inclusion criteria for the reference population were as follows: full-term normal delivery singletons without any thyroid-related diseases or family history of thyroid-related diseases, and without other conditions or medications that would cause changes in thyroid function-related indicator levels (TSH, FT3, FT4, T3, and T4). The exclusion criteria were as follows: Children with known thyroid disease, chronic illness (endocrine, inflammatory, autoimmune, cancer, and kidney diseases), thyroid medication usage (levothyroxine or growth hormone), or who had a mother with a thyroid-related disease.

\section{Laboratory tests}

Approximately 1-4 mL blood was drawn from the median cubital vein, and the serum was separated by a Roche Cobas P612 preprocessing system (Roche Diagnostics, Germany) within $6 \mathrm{~h}$. A Mindray automated chemiluminescence immunoassay analyzer CL-6000i (Mindray, China) was used to detect serum TSH, FT3, FT4, T3, and T4 levels. All test reagents were provided by Shenzhen Mindray Biomedical Electronics Co., Ltd. (Mindray, China). FT3 was converted from $\mathrm{pg} / \mathrm{mL}$ to $\mathrm{pmol} / \mathrm{L}$ by multiplying by 1.536 . FT4 was converted from $\mathrm{ng} / \mathrm{dL}$ to $\mathrm{pmol} / \mathrm{L}$ by multiplying by 12.872 . T3 was converted from $\mathrm{ng} / \mathrm{mL}$ to $\mathrm{nmol} / \mathrm{L}$ by multiplying by 1.536 . T4 was converted from $\mu \mathrm{g} / \mathrm{dL}$ to $\mathrm{nmol} / \mathrm{L}$ by multiplying by 12.872 .

\section{Statistical analysis}

$\mathrm{R}$ program version 3.6.3 and $\mathrm{R}$ studio version 1.4.1106 ( $\mathrm{R}$ Foundation for Statistical Computing, Vienna, Austria) were used for the statistical analyses. Categorical variables are described as frequencies and percentages. The ShapiroWilk test and skewness/kurtosis tests were used to check for normal distribution, and the Kruskal-Wallis test was applied to determine the gender differences in thyroid function distribution. The Goodman-Kruskal gamma coefficient was calculated for the distribution test in age group* gender crosstabs. Probability density curves within different populations (male, female, total) of all thyroid function indexes were presented using the "ggplott2" package. Scatter plots with linear fitting for the distribution of all thyroid indexes at different ages were constructed using GraphPad Prism Version 8.00 (GraphPad Software, CA, USA). All thyroid function indicators were presented as the $2.5^{\text {th }}$ percentile $(\mathrm{P} 2.5)$, median $(\mathrm{P} 50)$, and $97.5^{\text {th }}$ percentile (P97.5). All $\mathrm{P}$ values were two-sided, with $\mathrm{P}<0.05$ considered statistically significant.

\section{Results}

\section{Baseline characteristics of the study participants}

The main characteristics of the study participants are shown in Table 1. In the overall study, 645 children were male $(50.43 \%)$, and 634 children were female $(49.57 \%)$, and this proportion was considered acceptable. In terms of age distribution, $15.25 \%$ were aged $1-31$ days, $17.59 \%$ were aged 1-12 months, $15.56 \%$ were aged $12-36$ months, $15.79 \%$ were aged $36-72$ months, $19.39 \%$ were aged 72-108 months, and $16.42 \%$ were aged $108-144$ months. The numbers and proportion of the 72-108 months group 
Table 1 Comparison of age distribution between males and females $(\mathrm{G}, \mathrm{P})$

\begin{tabular}{lcccc}
\hline Age group & Total, $\mathrm{n}(\%)$ & Male, $\mathrm{n}(\%)$ & Female, $\mathrm{n}(\%)$ & $\mathrm{G}$ \\
\hline $1-31(\mathrm{~d})$ & $195(15.25)$ & $102(15.81)$ & $93(14.67)$ & -0.042 \\
$1-12(\mathrm{~m})$ & $225(17.59)$ & $112(17.36)$ & $113(17.82)$ & 0.273 \\
$12-36(\mathrm{~m})$ & $199(15.56)$ & $104(16.12)$ & $95(14.98)$ \\
$36-72(\mathrm{~m})$ & $202(15.79)$ & $108(16.74)$ & $94(14.83)$ \\
$72-108(\mathrm{~m})$ & $248(19.39)$ & $122(18.91)$ & $126(19.87)$ \\
$108-144(\mathrm{~m})$ & $210(16.42)$ & $97(15.04)$ & $113(17.82)$ \\
Total & $1,279(100.00)$ & $645(50.43)$ & $634(49.57)$ \\
\hline
\end{tabular}

Variables are presented as numbers (percentages). G represents the Goodman-Kruskal's Gamma test.
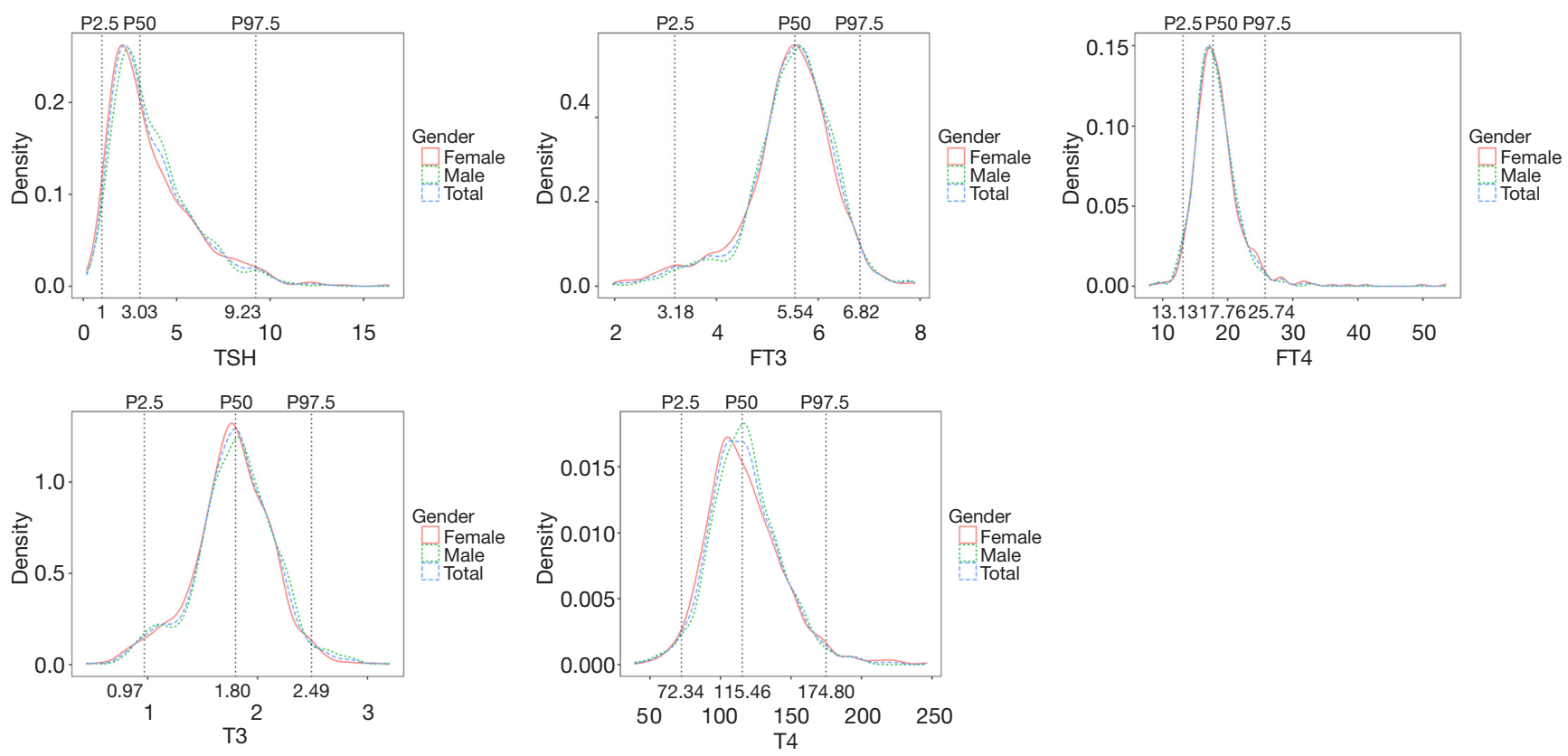

Figure 1 The probability density distributions of TSH, FT3, FT4, T3, and T4 by sex. The red, green, and blue curves represent the female, male and total distributions, respectively. The center line represents the P50, and the left and right lines represent the P2.5 and P97.5, respectively. TSH, thyroid-stimulating hormone; FT3, free triiodothyronine; FT4, free thyroxine; T3, total triiodothyronine; T4, total thyroxine; $\mathrm{P} 2.5,2.5^{\text {th }}$ percentile; $\mathrm{P} 50$, median; P97.5, $97.5^{\text {th }}$ percentile.

were slightly higher, but the age distribution differences within the male and female groups were not statistically significant $(\mathrm{G}=-0.042, \mathrm{P}=0.273$, Table 1).

\section{Distribution characteristics of thyroid hormones}

The distribution of serum TSH, FT3, FT4, T3, and T4 levels was analyzed on a linear scale in the total, male, and female populations (Figure 1). None of the five indicators were normally distributed, and thus the data were presented as binary percentiles (P2.5-P97.5). The reference range limits for TSH, FT3, T3, and T4 of males differed significantly from those of females $(\mathrm{P}<0.05)$, except for the lower limit of FT4, which did not vary significantly from the female equivalent in quantile testing $(\mathrm{P}=0.483)$ (Table 2). For FT3 and T3, the reference range for males was higher than females, and the reference range of $\mathrm{T} 4$ for males was narrower and higher than for females 
Table 2 Serum TSH, FT3, FT4, T3, and T4 levels according to gender in the study population

\begin{tabular}{|c|c|c|c|c|c|c|c|c|c|c|}
\hline Variables & \multicolumn{3}{|c|}{ Total } & \multicolumn{3}{|c|}{ Male } & \multicolumn{3}{|c|}{ Female } & $\mathrm{P}$ \\
\hline TSH (mlU/L) & 1.00 & 3.03 & 9.23 & 0.99 & 3.12 & 9.17 & 1.04 & 2.97 & 9.49 & $<0.001$ \\
\hline FT3 (pmol/L) & 3.18 & 5.54 & 6.82 & 3.37 & 5.58 & 6.86 & 3.11 & 5.51 & 6.81 & 0.024 \\
\hline FT4 (pmol/L) & 13.13 & 17.76 & 25.74 & 13.02 & 17.76 & 25.10 & 13.24 & 17.83 & 28.06 & 0.483 \\
\hline $\mathrm{T} 4$ (nmol/L) & 72.34 & 115.46 & 174.80 & 72.33 & 116.75 & 171.60 & 72.31 & 113.47 & 176.27 & 0.011 \\
\hline
\end{tabular}

Data were analyzed by the Kruskal-Wallis test. TSH, thyroid-stimulating hormone; FT3, free triiodothyronine; FT4, free thyroxine; T3, total triiodothyronine; T4, total thyroxine; P2.5, $2.5^{\text {th }}$ percentile; P50, median; P97.5, $97.5^{\text {th }}$ percentile.
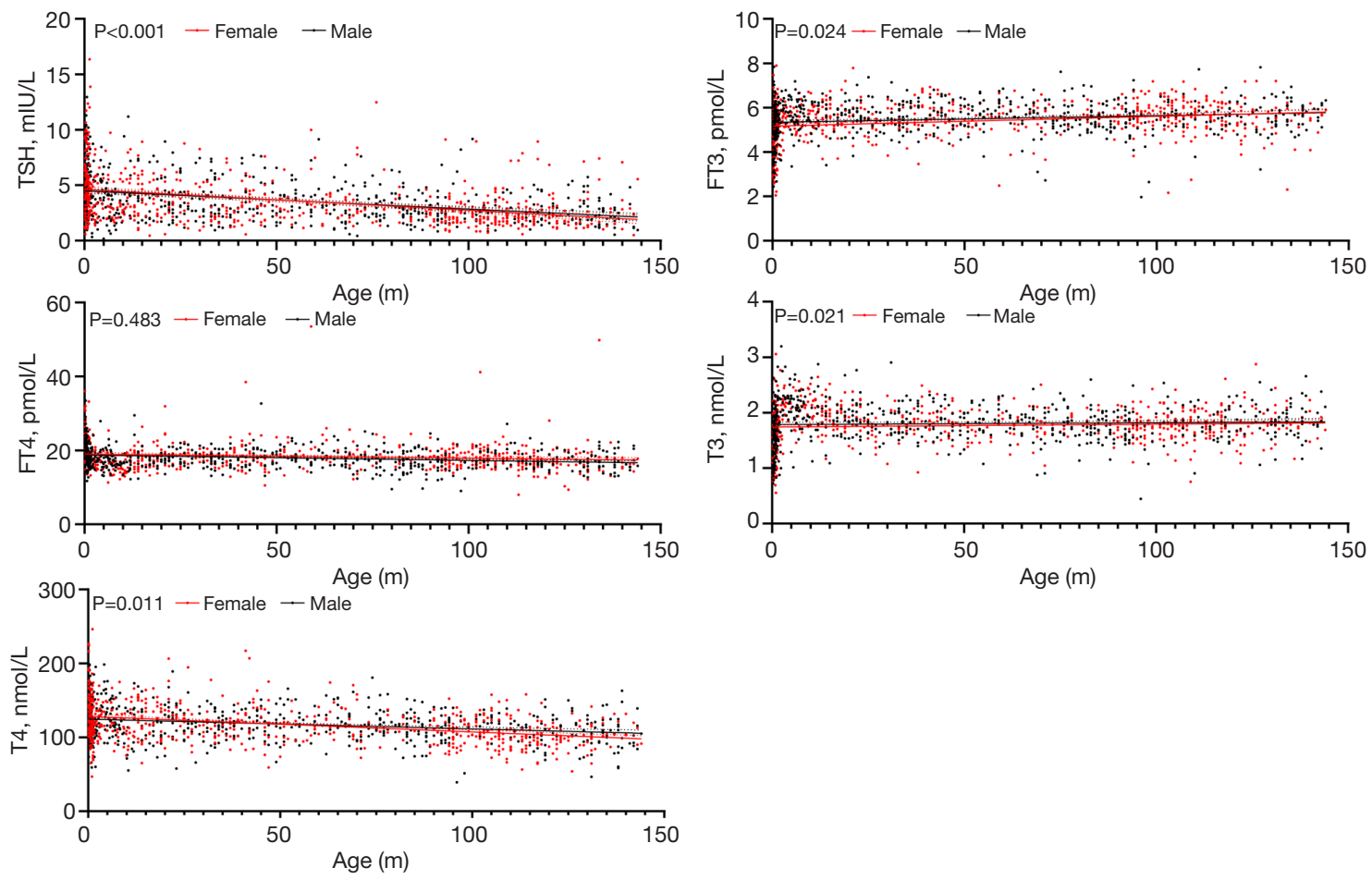

Figure 2 The scatter plot of TSH, FT3, FT4, T3, and T4 by age. The red and black dots represent the female and male values, respectively. The fitted curve based on a linear regression model adjusted for age represents the linear distribution of TSH, FT3, FT4, T3, and T4, and the red and black curves represent females and males, respectively. TSH, thyroid-stimulating hormone; FT3, free triiodothyronine; FT4, free thyroxine; T3, total triiodothyronine; T4, total thyroxine.

(P2.5-P97.5: 72.33-171.60 vs. 72.31-176.27 nmol/L; P50: 116.75 vs. $113.47 \mathrm{nmol} / \mathrm{L}, \mathrm{P}=0.011)$. The gender- and age-specific distribution of serum TSH, FT3, FT4, T3, and $\mathrm{T} 4$ levels also confirmed the difference in reference ranges between males and females (Figure 2). Moreover, serum TSH, FT3, FT4, T3, and T4 levels displayed wide variations during the first month of life and gradually narrowed with increasing age. For example, the reference range of serum TSH levels of each age group was 1.21-10.71, 0.94-9.74, 0.97-7.94, 1.11-7.71, 0.85-6.74, and 1.00-7.21 mIU/L, respectively (Table 3). In addition, in the serum TSH and T4 distribution, there was an evident shift toward lower levels with an increase in age, which specifically decreased faster in females than males. FT3 
Table 3 Serum TSH, FT3, FT4, T3, and T4 levels according to age in the study population

\begin{tabular}{lccccc}
\hline Age group & TSH $(\mathrm{mlU} / \mathrm{L})$ & FT3 $(\mathrm{pmol} / \mathrm{L})$ & FT4 $(\mathrm{pmol} / \mathrm{L})$ & T3 $(\mathrm{nmol} / \mathrm{L})$ & T4 $(\mathrm{nmol} / \mathrm{L})$ \\
\hline $1-31(\mathrm{~d})$ & $4.96[1.21,10.71]$ & $4.87[2.63,7.03]$ & $20.08[13.76,30.83]$ & $1.49[0.78,2.32]$ & $124.86[72.86,197.33]$ \\
$1-12(\mathrm{~m})$ & $3.79[0.94,9.74]$ & $5.54[3.31,6.72]$ & $17.25[13.13,24.16]$ & $1.98[1.00,2.75]$ & $119.58[71.99,175.63]$ \\
$12-36(\mathrm{~m})$ & $3.28[0.97,7.94]$ & $5.53[3.84,7.02]$ & $18.02[13.59,24.33]$ & $1.86[1.24,2.41]$ & $118.81[74.79,176.22]$ \\
$36-72(\mathrm{~m})$ & $3.00[1.11,7.71]$ & $5.72[3.67,6.80]$ & $18.09[13.14,25.53]$ & $1.83[1.09,2.33]$ & $116.62[76.52,170.20]$ \\
$72-108(\mathrm{~m})$ & $2.42[0.85,6.74]$ & $5.58[4.27,6.94]$ & $17.47[12.13,24.15]$ & $1.77[1.17,2.34]$ & $110.89[74.79,152.29]$ \\
$108-144(\mathrm{~m})$ & $2.20[1.00,7.21]$ & $5.64[3.94,7.12]$ & $17.12[12.65,23.16]$ & $1.81[1.15,2.43]$ & $102.40[61.50,149.49]$ \\
\hline
\end{tabular}

The P50 [P2.5, P97.5] for each thyroid hormone in six age groups is presented. TSH, thyroid-stimulating hormone; FT3, free triiodothyronine; FT4, free thyroxine; T3, total triiodothyronine; T4, total thyroxine; P50, median; P2.5, 2.5 ${ }^{\text {th }}$ percentile; P97.5, 97.5 percentile.
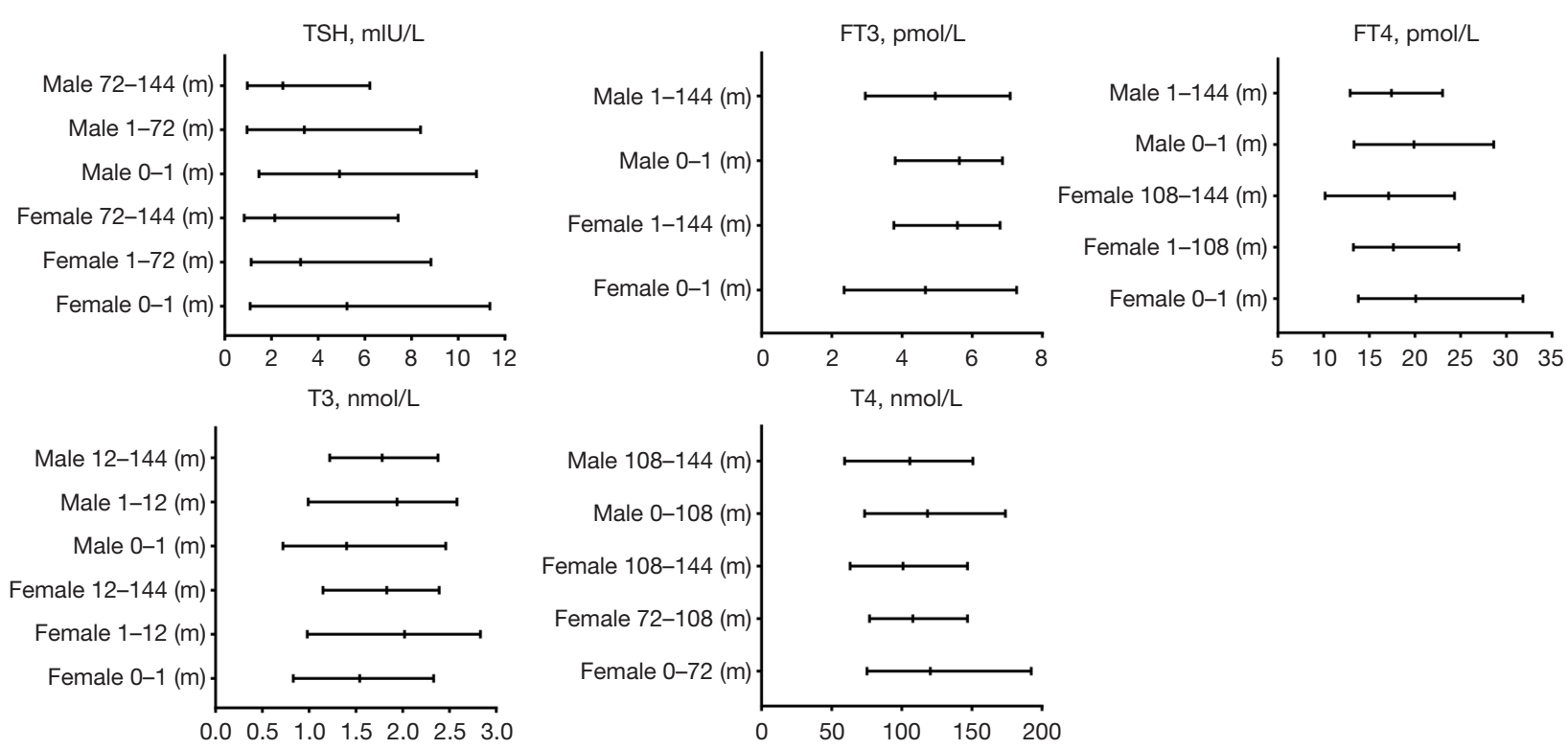

Figure 3 The grouping distributions of the RIs stratified by age and sex for TSH, FT3, FT4, T3, and T4. The line exhibits the 95\% interval range, and the three short vertical lines represent the P2.5, P50, and P97.5 from left to right. RI, reference interval; TSH, thyroidstimulating hormone; FT3, free triiodothyronine; FT4, free thyroxine; T3, total triiodothyronine; T4, total thyroxine; P2.5, $2.5^{\text {th }}$ percentile; P50, median; P97.5, $97.5^{\text {th }}$ percentile.

gradually returned towards the P50 (Table 2) after the first month of life and remained constant in both sexes (Figure 2).

\section{Pediatric RIs for thyroid function}

Differences in serum TSH, FT3, FT4, T3, and T4 levels were observed in different age groups according to the Kruskal-Wallis analysis and Nemenyi test. The RIs for thyroid hormones in specific age and sex groups were calculated based on the results described above (Figure 3).
The $\mathrm{P} 50$ and $95 \%$ distributions (P2.5-P97.5) of serum TSH, FT3, FT4, T3, and T4 in pediatric participants are presented in Table 4. The distributions of TSH RI were much wider during the first month and gradually became narrower with increasing age in both sexes. The FT3 RI for females gradually narrowed after the first month, but the opposite effect was seen in males. The FT4 RI displayed a similar trend and distribution for males and females during the first month of life, but the FT4 RI for females showed a decreasing shift in the 9- to 12-year age group. The RIs 
Table 4 Sex- and age-specific pediatric RIs for TSH, FT3, FT4, $\mathrm{T} 3$, and $\mathrm{T} 4$

\begin{tabular}{|c|c|c|c|}
\hline $\begin{array}{l}\text { Thyroid } \\
\text { hormones }\end{array}$ & Groups & Age group (m) & RI, P50 [P2.5, P97.5] \\
\hline \multirow[t]{6}{*}{ TSH (mlU/L) } & Female & $0-1$ & $5.23[1.08,11.35]$ \\
\hline & & $1-72$ & $3.24[1.14,8.83]$ \\
\hline & & $72-144$ & $2.14[0.83,7.42]$ \\
\hline & Male & $0-1$ & $4.91[1.46,10.78]$ \\
\hline & & $1-72$ & $3.40[0.95,8.38]$ \\
\hline & & $72-144$ & $2.48[0.96,6.21]$ \\
\hline \multirow[t]{4}{*}{ FT3 (pmol/L) } & Female & $0-1$ & $4.67[2.35,7.27]$ \\
\hline & & $1-144$ & $5.58[3.77,6.80]$ \\
\hline & Male & $0-1$ & $4.95[2.96,7.08]$ \\
\hline & & $1-144$ & $5.64[3.81,6.86]$ \\
\hline \multirow[t]{5}{*}{ FT4 (pmol/L) } & Female & $0-1$ & $20.08[13.82,31.83]$ \\
\hline & & $1-108$ & $17.63[13.26,24.80]$ \\
\hline & & $108-144$ & $17.12[10.16,24.34]$ \\
\hline & Male & $0-1$ & $19.89[13.34,28.65]$ \\
\hline & & $1-144$ & $17.43[12.90,23.04]$ \\
\hline \multirow[t]{6}{*}{ T3 (nmol/L) } & Female & $0-1$ & $1.40[0.72,2.46]$ \\
\hline & & $1-12$ & $1.94[0.99,2.58]$ \\
\hline & & $12-144$ & $1.78[1.22,2.38]$ \\
\hline & Male & $0-1$ & $1.54[0.83,2.33]$ \\
\hline & & $1-12$ & $2.02[0.98,2.83]$ \\
\hline & & $12-144$ & $1.83[1.15,2.39]$ \\
\hline \multirow[t]{5}{*}{ T4 (nmol/L) } & Female & $0-72$ & $120.48[75.28,192.48]$ \\
\hline & & $72-108$ & $107.87[77.03,146.91]$ \\
\hline & & $108-144$ & $100.92[63.29,146.94]$ \\
\hline & Male & $0-108$ & $118.36[73.63,173.85]$ \\
\hline & & $108-144$ & $105.68[59.31,150.72]$ \\
\hline
\end{tabular}

TSH, thyroid-stimulating hormone; FT3, free triiodothyronine; FT4, free thyroxine; T3, total triiodothyronine; T4, total thyroxine; $\mathrm{RI}$, reference interval; P50, median; P2.5, $2.5^{\text {th }}$ percentile; P97.5, $97.5^{\text {th }}$ percentile.

of T3 and T4 displayed a similar gradual increase and concentration trend in both sexes.

\section{Discussion}

The application of RIs for conventional indicators has been well established in adult and even gestation-specific populations (24). However, accurate age- and sex-specific RIs are not readily available in pediatric laboratory medicine and have been largely overlooked (26). Several national and global initiatives have begun to close the critical evidence gap in pediatric RIs. For example, in 2012, the CALIPER study was the first to publish age- and sex-specific RIs for 40 common biochemical indicators in Canadian children (1 day to 19 years) (27). In 2021, the National Health Commission of China released the RIs for 31 biochemistry and blood cell parameters in Chinese children (from 28 days to 18 years) (http://www.nhc.gov.cn, WS/T7792021, and WS/T780-2021). Thyroid function tests are among the most requested in pediatric care to prevent physical and developmental impairments. The current study establishes age- and sex-specific RIs for thyroid hormones, including TSH, FT3, FT4, T3, and T4 for a Chinese pediatric population consisting of 1,279 healthy participants (aged 1 day to 14 years) on the Mindray automated chemiluminescence immunoassay analyzer CL-6000i.

To date, CALIPER has established age- and sex-specific RIs of thyroid hormones on the Abbott ARCHITECT c8000 (27), Ortho VITROS (17), Beckman Coulter (28), and Roche Cobas 8000 e602 (12) systems. While age and sex patterns are very similar between studies, the detailed RIs (e.g., TSH) of each subpopulation show significant differences within some analytes due to inter-assay differences. RIs of thyroid hormones have reportedly been associated with iodine uptake, child age, sex, ethnicity, and various detection methods or analyzers $(15,29)$. For example, FT4 concentration levels are lowest in Dutch children and highest in children of non-Western or Surinamese origin (15). FT3 significantly increased upon puberty and showed a significant sex-specific difference at which age, while FT4 remained constant throughout puberty (10). In China, the sex/geographic/assay-specific and gestation-specific RIs of thyroid hormones have been established in adult populations (22-25), emphasizing the need for assay-specific RIs of thyroid hormones in Chinese pediatric populations to avoid false positive or negative results.

Similar to CALIPER and other internationally recognized studies, a partition within the first month of life is required to establish hormone RIs due to the dynamic fluctuations of the post-parturition period (30). The RIs of TSH, FT3, FT4, T3, and T4 established in the present study displayed wide variation during the first month of life and then gradually narrowed with increasing age in both sexes. The trend displayed across pediatric age was consistent with results obtained by CALIPER on the 
Roche Cobas 8000 e602 system (12), but the Beckman Coulter system showed no differences in age $<1$ year (28). A significant sex-specific difference was observed on the Ortho VITROS and Beckman Coulter systems, but not the Roche Cobas 8000 e602 system in studies conducted by CALIPER. In this study, serum TSH, FT3, T3, and T4 showed significant sex-specific differences, but not FT4. Furthermore, the range of age partitioning confirmed by statistical methods was also different amongst these analyzers. The medians of each thyroid hormone in whole pediatric populations detected by Mindray CL-6000i were comparable to those found by other analyzers, like Roche Cobas 8000 e602, e601, Ortho VITROS, and the Beckman Coulter systems $(12,15,17,28)$. Still, the RIs in each partitioning group established by Mindray CL-6000i were moderately narrower or wider than the previously published RIs (e.g., TSH: 1.23-27.2 mIU/L for e602 vs. $1.08-$ $11.35 \mathrm{mIU} / \mathrm{L}$ for CL-6000i in females $<1$ month).

Detection assay is an important determinant of the large differences in RI establishment for thyroid hormones. Besides the widely used immunoassay, liquid chromatography-mass spectrometry and/or equilibrium dialysis are also used in some analyzers $(31,32)$. the Mindray automated chemiluminescence immunoassay analyzer CL-6000i detected thyroid hormones by integrating the advantages of immunoassay and chemiluminescence methods, and is the widely used instrument in mainland of China. This highlights the lack of standardization for thyroid hormones and the need for assay-specific RI establishment of CL-6000i analyzer. However, establishing RIs using binary percentiles may not accurately depict the dynamics of certain biomarkers occurring alongside pediatric physiological development due to the inherent influence of population size and limited statistical power. Moreover, the detachment from the placenta and the change in thermal environment lead to a rapid change in the thyroid hormones of neonates (33), and baseline levels of thyroid hormones show differences in preterm infants, same-sex twins, singletons, and differentsex twins within the first month of life (34-36). Same-sex twins and preterm infants have a high incidence of $\mathrm{CH}$ but also a high probability of being missed at newborn screening. In future, the establishment and diagnostic rigor of binary vs. continuous RIs for clinical decision-making, particularly during the first month of life, is urgently needed.

\section{Conclusions}

In conclusion, this study establishes RIs for TSH, FT3,
FT4, T3, and T4 on the Mindray CL-6000i analytical platform that will allow laboratories using this system to interpret TSH assay results based on accurate RIs. As recommended by the CLSI EP28-A3c guidelines, RIs should be validated for each clinical laboratory's local pediatric population and individual analyzer before implementation.

\section{Acknowledgments}

Funding: This work was supported by the Natural Science Foundation of Wuhan Municipal Health Commission (grant nos. WX18Q27, WX18Q03, WX21M03), the Natural Science Foundation of Hubei Municipal Health Commission (grant no. WJ2021M016), and The Top Medical Young Talents of Hubei Province (2016).

\section{Footnote}

Reporting Checklist: The authors have completed the STROBE reporting checklist. Available at https://dx.doi. org/10.21037/tp-21-389

Data Sharing Statement: Available at https://dx.doi. org/10.21037/tp-21-389

Conflicts of Interest: All authors have completed the ICMJE uniform disclosure form (available at https://dx.doi. org/10.21037/tp-21-389). CX and GS report that they are from Shenzhen Mindray Bio-Medical Electronics Co., Ltd., Shenzhen, China. The other authors have no conflicts of interest to declare.

Ethical Statement: The authors are accountable for all aspects of the work in ensuring that questions related to the accuracy or integrity of any part of the work are appropriately investigated and resolved. The study was conducted in accordance with the Declaration of Helsinki (as revised in 2013) for research on human participants, and the study protocols were approved by the Ethics Committee and Institutional Review Board of the Wuhan Children's Hospital (Wuhan Maternal and Child Healthcare Hospital) (2020R052). Written informed consent was obtained from all pediatric participants (provided by at least a parent or guardian) under physical examination.

Open Access Statement: This is an Open Access article distributed in accordance with the Creative Commons 
Attribution-NonCommercial-NoDerivs 4.0 International License (CC BY-NC-ND 4.0), which permits the noncommercial replication and distribution of the article with the strict proviso that no changes or edits are made and the original work is properly cited (including links to both the formal publication through the relevant DOI and the license). See: https://creativecommons.org/licenses/by-nc-nd/4.0/.

\section{References}

1. Al-Suhaimi EA, Al-Khater K. Functions of stem cells of thyroid glands in health and disease. Rev Endocr Metab Disord 2019;20:187-95.

2. Fekete C, Lechan RM. Central regulation of hypothalamic-pituitary-thyroid axis under physiological and pathophysiological conditions. Endocr Rev 2014;35:159-94.

3. Hanley P, Lord K, Bauer AJ. Thyroid disorders in children and adolescents: a review. JAMA Pediatr 2016;170:1008-19.

4. Leung AKC, Leung AAC. Evaluation and management of the child with hypothyroidism. World J Pediatr 2019;15:124-34.

5. Srinivasan S, Misra M. Hyperthyroidism in children. Pediatr Rev 2015;36:239-48.

6. Chaudhari M, Slaughter JL. Thyroid function in the neonatal intensive care unit. Clin Perinatol 2018;45:19-30.

7. Wassner AJ. Congenital hypothyroidism. Clin Perinatol 2018;45:1-18.

8. van der Spek AH, Fliers E, Boelen A. Thyroid hormone metabolism in innate immune cells. J Endocrinol 2017;232:R67-r81.

9. Jabbar A, Pingitore A, Pearce SH, et al. Thyroid hormones and cardiovascular disease. Nat Rev Cardiol 2017;14:39-55.

10. Shivaraj G, Prakash BD, Sonal V, et al. Thyroid function tests: a review. Eur Rev Med Pharmacol Sci 2009;13:341-9.

11. Lazarus J, Brown RS, Daumerie C, et al. 2014 European thyroid association guidelines for the management of subclinical hypothyroidism in pregnancy and in children. Eur Thyroid J 2014;3:76-94.

12. Bohn MK, Higgins V, Asgari S, et al. Paediatric reference intervals for 17 Roche cobas 8000 e602 immunoassays in the CALIPER cohort of healthy children and adolescents. Clin Chem Lab Med 2019;57:1968-79.

13. Omuse G, Kassim A, Kiigu F, et al. Reference intervals for thyroid stimulating hormone and free thyroxine derived from neonates undergoing routine screening for congenital hypothyroidism at a university teaching hospital in Nairobi, Kenya: a cross sectional study. BMC Endocr Disord 2016;16:23.

14. Lem AJ, de Rijke YB, van Toor H, et al. Serum thyroid hormone levels in healthy children from birth to adulthood and in short children born small for gestational age. J Clin Endocrinol Metab 2012;97:3170-8.

15. Önsesveren I, Barjaktarovic M, Chaker L, et al. Childhood thyroid function reference ranges and determinants: a literature overview and a prospective cohort study. Thyroid 2017;27:1360-9.

16. Campbell PJ, Brown SJ, Kendrew P, et al. Changes in thyroid function across adolescence: a longitudinal study. J Clin Endocrinol Metab 2020;105:dgz331.

17. Higgins V, Fung AWS, Chan MK, et al. Pediatric reference intervals for 29 Ortho VITROS 5600 immunoassays using the CALIPER cohort of healthy children and adolescents. Clin Chem Lab Med 2018;56:327-40.

18. Boucai L, Surks MI. Reference limits of serum TSH and free $\mathrm{T} 4$ are significantly influenced by race and age in an urban outpatient medical practice. Clin Endocrinol (Oxf) 2009;70:788-93.

19. Yu L, Yue H, Lin H, et al. Reference interval in preliminary investigation of maternal thyroid function during pregnancy in Shenzhen China. Zhonghua Yi Xue Za Zhi 2014;94:3175-8.

20. Li ZZ, Yu BZ, Wang JL, et al. Reference intervals for thyroid-stimulating hormone and thyroid hormones using the access TSH 3rd IS method in China. J Clin Lab Anal 2020;34:e23197.

21. Lin X, Zheng LJ, Li HB, et al. Reference intervals for preterm thyroid function during the fifth to seventh day of life. Clin Biochem 2021. [Epub ahead of print]. doi: 10.1016/j.clinbiochem.2021.05.014.

22. Zou Y, Wang D, Cheng X, et al. Reference intervals for thyroid-associated hormones and the prevalence of thyroid diseases in the Chinese population. Ann Lab Med 2021;41:77-85.

23. Wang D, Yu S, Ma C, et al. Reference intervals for thyroid-stimulating hormone, free thyroxine, and free triiodothyronine in elderly Chinese persons. Clin Chem Lab Med 2019;57:1044-52.

24. Wang D, Yu S, Cheng X, et al. Nationwide Chinese study for establishing reference intervals for thyroid hormones and related tests. Clin Chim Acta 2019;496:62-7. Erratum in: Clin Chim Acta 2021;512:191.

25. Yang X, Meng Y, Zhang Y, et al. Thyroid function 
reference ranges during pregnancy in a large Chinese population and comparison with current guidelines. Chin Med J (Engl) 2019;132:505-11.

26. Adeli K, Higgins V, Trajcevski K, et al. The Canadian laboratory initiative on pediatric reference intervals: A CALIPER white paper. Crit Rev Clin Lab Sci 2017;54:358-413.

27. Colantonio DA, Kyriakopoulou L, Chan MK, et al. Closing the gaps in pediatric laboratory reference intervals: a CALIPER database of 40 biochemical markers in a healthy and multiethnic population of children. Clin Chem 2012;58:854-68.

28. Karbasy K, Lin DC, Stoianov A, et al. Pediatric reference value distributions and covariate-stratified reference intervals for 29 endocrine and special chemistry biomarkers on the Beckman Coulter Immunoassay Systems: a CALIPER study of healthy community children. Clin Chem Lab Med 2016;54:643-57.

29. Tahmasebi H, Trajcevski K, Higgins V, et al. Influence of ethnicity on population reference values for biochemical markers. Crit Rev Clin Lab Sci 2018;55:359-75

30. Mori A, Tuli G, Magaldi R, et al. Plasma cortisol and ACTH levels in $416 \mathrm{VLBW}$ preterm infants during the first month of life: distribution in the AGA/SGA

Cite this article as: Yao $\mathrm{C}, \mathrm{Wu} M$, Liu $M$, Chen $\mathrm{X}$, Zhu H, Xiong C, Wang D, Xiang Y, Suo G, Wang J, Sun H, Yuan C, Xia Y. Age- and sex-specific reference intervals for thyroid hormones in a Chinese pediatrics: a prospective observational study of 1,279 healthy children. Transl Pediatr 2021;10(10):2479-2488. doi: 10.21037/tp-21-389 population. J Perinatol 2019;39:934-40.

31. Soldin OP, Jang M, Guo T, et al. Pediatric reference intervals for free thyroxine and free triiodothyronine. Thyroid 2009;19:699-702.

32. La'ulu SL, Rasmussen KJ, Straseski JA. Pediatric reference intervals for free thyroxine and free triiodothyronine by equilibrium dialysis-liquid chromatography-tandem mass spectrometry. J Clin Res Pediatr Endocrinol 2016;8:26-31.

33. Eng L, Lam L. Thyroid function during the fetal and neonatal periods. Neoreviews 2020;21:e30-6.

34. Jiang X, Huang YL, Feng Y, et al. Same-sex twins have a high incidence of congenital hypothyroidism and a high probability to be missed at newborn screening. Clin Chim Acta 2020;502:111-5.

35. Medda E, Vigone MC, Cassio A, et al. Neonatal screening for congenital hypothyroidism: what can we learn from discordant twins? J Clin Endocrinol Metab 2019;104:5765-79.

36. Vigone MC, Caiulo S, Di Frenna M, et al. Evolution of thyroid function in preterm infants detected by screening for congenital hypothyroidism. J Pediatr 2014;164:1296-302.

(English Language Editor: D. Fitzgerald) 